\title{
J. VIGNES
}

\section{Implémentation des méthodes d'optimisation : test d'arrêt optimal, contrôle et précision de la solution (II)}

Revue française d'automatique, d'informatique et de recherche opérationnelle. Recherche opérationnelle, tome 18, n 2 (1984), p. 103-129.

<http://www.numdam.org/item?id=RO_1984_18_2_103_0>

(C) AFCET, 1984, tous droits réservés.

L'accès aux archives de la revue « Revue française d'automatique, d'informatique et de recherche opérationnelle. Recherche opérationnelle » implique l'accord avec les conditions générales d'utilisation (http://www.numdam.org/ legal.php). Toute utilisation commerciale ou impression systématique est constitutive d'une infraction pénale. Toute copie ou impression de ce fichier doit contenir la présente mention de copyright.

\section{Numdam}

Article numérisé dans le cadre du programme

Numérisation de documents anciens mathématiques

http://www.numdam.org/ 


\title{
IMPLEMENTATION \\ DES MÉTHODES D'OPTIMISATION : TEST D'ARRÊT OPTIMAL, CONTRÔLE ET PRÉCISION DE LA SOLUTION (II) (*)
}

\author{
par J. Vignes $\left({ }^{1}\right)$
}

\footnotetext{
Abstract. - Lorsque l'on implémente sur ordinateur des algorithmes d'optimisation, plusieurs problèmes se posent:

- comment arrêter le processus itératif:

- comment s'assurer que la solution obtenue est informatiquement satisfaisante et la meilleure que puisse fournir la machine;

- comment conclure que la solution informatique est une approximation de la solution mathématique et quelle est sa précision.

Nous présentons en deux articles des méthodes originales permettant de résoudre ces problèmes. Le premier article, paru dans le numéro précédent de la Revue, traitait des méthodes d'implémentation. Nous présentons ici les logiciels correspondants.
}

Keywords: Optimisation; analyse des erreurs d'arrondi; critère d'arrêt optimal; précision de résultats d'algorithmes; validité des logiciels numériques.

Résumé. - In implementing any optimization method on a computer, several problems arise:

- how to break off the iterative process;

- how to determine the satisfactory computed solution and be sure the computer cannot provide a better one;

- how to tell whether or not the computed solution approximates the mathematical solution, and to what degree of accuracy.

New methods for solving these problems appeared in the preceding issue of this Journal. The corresponding software are presented here.

Mots clés : Optimization; round-off error analysis; optimal termination criterion; accuracy in the results of computations; validity of numerical software.

\section{INTRODUCTION}

Pour pouvoir mettre en ceuvre les méthodes décrites dans la première partie de cet article, il faut pouvoir faire faire automatiquement par la machine l'analyse de la propagation des erreurs d'arrondi due à l'arithmétique virgule flottante et pouvoir évaluer, pour toute valeur issue du calcul de n'importe quelle fonctionnelle, son nombre de chiffres décimaux significatifs exacts. Il

(*) Reçu en juin 1982.

( ${ }^{1}$ ) Professeur à l'Institut de Programmation de l'Université Pierre-et-Marie-Curie de Paris. Conseiller Scientifique à l'Institut Français du Pétrole.

R.A.I.R.O. Recherche opérationnelle/Operations Research, 0399-0559/84/02 103 27/\$4.70

(C) AFCET-Bordas-Dunod 
faut donc disposer d'un logiciel de Permutation-Perturbation permettant, d'une part, de faire exécuter le calcul de toute fonctionnelle en faisant changer l'ordre des opérations et, d'autre part, d'affecter au résultat de toute opération arithmétique une valeur soit par excès soit par défaut. C'est ce logiciel et sa mise en cuvre que nous allons décrire dans le paragraphe suivant.

\section{LE LOGICIEL DE PERMUTATION-PERTURBATION}

\section{1. La permutation}

Nous avons vu que dans la pratique il suffit de permuter les formules linéaires. Ainsi, pour réaliser les permutations, c'est-à-dire changer l'ordre d'exécution des opérations, il suffit de décomposer la fonctionnelle dont on veut estimer le nombre de chiffres décimaux significatifs exacts du résultat en une somme algébrique de monômes. Ces monômes sont rangés dans un vecteur $P$ de dimension $N, N$ étant le nombre de monômes.

Soit $F$ cette fonctionnelie; elle se présente sous la forme :

$$
F=\sum_{i=1}^{N} P_{i}
$$

Avant chaque exécution du calcul de $F$, ces $N$ monômes $P_{i}$ sont rangés dans un ordre aléatoire établi par un sous-programme de génération de nombres aléatoires dans le vecteur $P$, comme il est montré dans la fonction PEPER donné en annexe. Ainsi, lors des trois calculs nécessaires de $F$ pour estimer son nombre de chiffres décimaux significatifs, le calcul de la somme des monômes se fait-il chaque fois dans un ordre différent.

Exemple. - Soit à chercher le minimum de la fonction :

$$
\left.\begin{array}{c}
F=f_{1}^{2}+f_{2}^{2} \\
f_{1}=7 X_{1}^{2}+3 X_{1} X_{2}+4 X_{1}-X_{2}-41, \\
f_{2}=10 X_{1}^{2}+4 X_{1} X_{2}+5 X_{1}-2 X_{2}-56
\end{array}\right\}
$$

par la méthode présentée dans la première partie, qui permet de minimiser les fonctionnelles se présentant sous la forme d'une somme de carrés.

Il est nécessaire d'écrire le sous-programme qui calcule $F$ et grad $F$ sous la forme présentée en annexe qui consiste à décomposer $F$ et $\operatorname{grad} F$ en une somme de monômes. 


\section{2. La perturbation}

La perturbation consiste à fournir comme résultat de toute opération arithmétique soit sa valeur par défaut soit sa valeur par excès. Considérons l'opération arithmétique définie par :

$$
R=X \omega Y, \quad X, Y, R \in \mathbb{F},
$$

$\omega$ étant un opérateur informatique quelconque.

$R^{-}$représente la valeur par défaut et $R^{+}$la valeur par excès du résultat $R$. Le résultat exact $r \in \mathbb{R}$, inaccessible en informatique, est toujours compris entre $R^{-}$et $R^{+}$:

$$
R^{-} \leqq r \leqq R^{+}
$$

Lorsque l'ordinateur travaille en arithmétique tronquée virgule flottante normalisée avec $p$ bits de mantisse, le résultat de toute opération arithmétique est toujours $R^{-}$(par défaut). Aussi, pour obtenir $R^{+}$doit-on ajouter 1 au dernier bit de la mantisse.

Lorsque l'ordinateur travaille en arithmétique arrondie, le résultat de toute opération arithmétique est soit par défaut soit par excès. Aussi doit-on ajouter ou retrancher 1 au dernier bit de la mantisse ou ne rien faire (ajouter 0 ) pour obtenir les valeurs par défaut ou par excès.

Le logiciel de perturbation consiste donc :

- dans le cas de l'arithmétique tronquée, à ajouter aléatoirement un 0 ou un 1 au dernier bit de la mantisse, les 0 et les 1 étant fournis par un générateur de nombres aléatoires uniformément répartis dans l'intervalle [0,2[ et en en prenant la partie entière;

- dans le cas de l'arithmétique arrondie, à ajouter $-1,0$ ou +1 au dernier bit de la mantisse. Les valeurs $-1,0$ et +1 sont fournies par un générateur de nombres aléatoires uniformément répartis dans l'intervalle ]$-2,+2[$ et en en prenant la partie entière.

Nous considérons, pour expliquer le logiciel permettant d'ajouter ou de retrancher 1 au dernier bit de la mantisse, le cas simple d'un ordinateur travaillant en arithmétique binaire normalisée avec des mots de $n$ bits codés de la façon suivante : 1 bit pour le signe ( 0 pour les nombres positifs et 1 pour les négatifs), $m$ bits réservés à la caractéristique (exposant + biais) et $p$ bits de mantisse. Nous supposons en outre que l'arithmétique est en troncature. Ainsi, tout élément $X \in \mathbb{F}$ représentant $x \in \mathbb{R}$ représente la valeur par 
défaut de $x$, soit $X^{-}$'Lorsqu'on ajoute un 1 au dernier bit de la mantisse, la valeur obtenue est $X^{+}$, valeur par excès de $x$ :

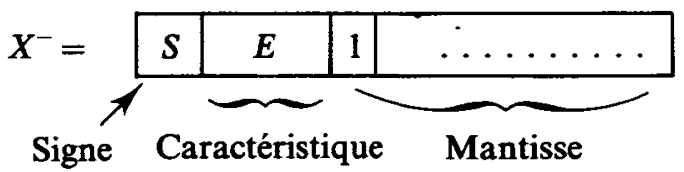

Pour obtenir $X^{+}$il faut ajouter à $X^{-}$le nombre $M 1$ défini par :

$$
M 1=\begin{array}{|l|l|lll|}
\hline S & E & 0 & 0 \ldots \ldots \ldots \ldots & \ldots \\
\hline
\end{array}
$$

Pour obtenir $M 1$ on peut écrire une procédure soit en langage d'assemblage, soit en langage Fortran qui réalise les opérations booléennes suivantes :

et

$$
\left.\begin{array}{c}
M 1=\left(X^{-} \cap M 2\right) \cup M 3 \\
X^{+}=X^{-}+M 1,
\end{array}\right\}
$$

$M 2$ et $M 3$ étant les masques définis par :

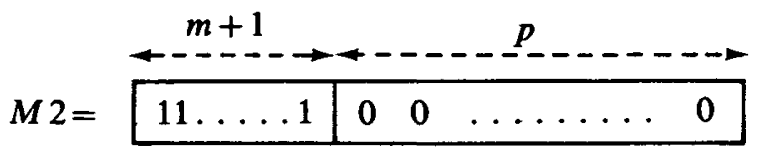

$$
\begin{aligned}
& M 3=\begin{array}{r|rrr}
00 \ldots 0 & 0 & \ldots \ldots \ldots & 01 \\
\hline
\end{array}
\end{aligned}
$$

Dans le cas où la codification binaire des valeurs négatives se fait en complément, il faut, pour obtenir la valeur de $X^{+}$lorsque $X^{-}$est négatif, retrancher 1 au dernier bit de la mantisse de $X^{-}$et donc effectuer l'opération :

$$
X^{+}=X^{-}-M 1 \text {. }
$$

Nous donnons en annexe la fonction $X V$ qui permet de perturber sur CDC de la série des 6000 ou 7000 toute valeur $X$ positive ou négative.

Le logiciel PEPER donné en annexe permet donc de réaliser pour le calcul de toute fonctionnelle la permutation et la perturbation. Ce logiciel est portable sur tout ordinateur; seule la fonction $X V$ à laquelle il fait appel est spécifique de la machine utilisée, et doit donc être réécrite comme il a été expliqué ci-dessus. 


\section{ADAPTATION DU LOGICIEL PEPER AUX CODES D'OPTIMISATION}

\section{1. Structuration des fonctionnelles du problème à traiter}

Tout code général d'optimisation nécessite que l'utilisateur écrive sous forme de procédures (sous-programmes Fortran par exemple) les fonctionnelles du problème qu'il a à traiter :

- calcul de la fonctionnelle $F$ à optimiser,

- calcul de $\operatorname{grad} F$ ou $\|\operatorname{grad} F\|$,

- calcul des contraintes.

Pour utiliser le logiciel PEPER, il est nécessaire que chacune des fonctionnelles soit écrite sous la forme d'une suite de monômes, comme il est montré dans l'exemple fourni en annexe, qui permet de résoudre le système non linéaire d'ordre 2 défini par (2) en cherchant le minimum nul de :

$$
F=f_{1}^{2}+f_{2}^{2} \text {. }
$$

\section{2. Implémentation du test d'arrêt optimal dans les codes d'optimisation de fonctions non contraintes}

L'implémentation du test d'arrêt optimal dans tout code d'optimisation non contrainte est très aisée. En effet, elle consiste à remplacer le test d'arrêt classique par le calcul, à chaque itération $q$, du nombre de chiffres décimaux significatifs $C_{i}^{(q)}$ ou $K^{(q)}$ de chaque composante de $\operatorname{grad} F\left(X^{(q)}\right)$ ou de $\left\|\operatorname{grad} F\left(X^{(q)}\right)\right\|$

Pour réaliser cela, il suffit d'appeler trois fois la procédure calculant $\operatorname{grad} F\left(X^{(q)}\right)$ ou $\left\|\operatorname{grad} F\left(X^{(q)}\right)\right\|$ qui a été structurée comme précédemment expliqué et qui utilise le logiciel PEPER.

Ainsi obtient-on trois valeurs différentes de :

$$
\frac{\partial F_{j}\left(X^{q}\right)}{\partial x_{i}}, \quad i=1, \ldots, n, \quad j=1,2,3
$$

ou de

$$
\left\|\operatorname{grad} F_{j}\left(X^{(q)}\right)\right\|, \quad j=1,2,3 .
$$

On calcule ensuite la moyenne et l'écart-type de ces quantités et en utilisant l'équation (26) donnée dans la première partie de cet article, on en déduit les $C_{i}^{(q)}$ ou $K^{(q)}$.

Lorsque la méthode d'optimisation est convergente, les $C_{i}^{(q)}, i=1, \ldots, n$ ou $K^{(q)}$ sont décroissants et tendent vers zéro. 
Ainsi, lorsque tous les $C_{i}^{(q)}<1(i=1,2, \ldots, n)$ ou $K^{(q)}<1$, cela veut dire alors que les composantes de grad $F\left(X^{(q)}\right)$ ou $\left\|\operatorname{grad} F\left(X^{(q)}\right)\right\|$ doivent être considérées comme nulles et qu'une solution informatiquement satisfaisante est atteinte.

Le processus itératif doit par conséquent être interrompu.

Le test d'arrêt optimal ainsi défini est présenté en annexe dans le code d'optimisation proposé.

\section{3. Implémentation du test d'arrêt optimal dans les codes d'optimisation de fonctions contraintes}

Comme nous l'avons défini dans la première partie, les codes d'optimisation contrainte consistent à résoudre :

sous les contraintes :

$$
\underset{\text { Sup }}{\text { Inf }} F(X) \text {, }
$$

$$
\begin{array}{cc}
H_{i}(X)=0, & i=1, \ldots, m, \quad m \in \mathbb{N}, \\
G_{j}(X) \geqq 0, & j=m+1, \ldots, p, \quad p \in \mathbb{N} .
\end{array}
$$

Comme il est montré dans la première partie de cet article, le processus itératif doit être interrompu à l'itération $q$ lorsque $X^{(q)}$ satisfait les conditions de Kuhn et Tucker. Mais, pour s'assurer, à l'itération $q$, que les conditions de Kuhn et Tucker sont satisfaites, il faut que le code d'optimisation utilisé fournisse non seulement les valeurs du vecteur $X^{(q)}$ mais aussi celles des vecteurs des multiplicateurs de Lagrange $\lambda^{(q)}$ et $\mu^{(q)}$.

Pour vérifier les conditions de Kuhn et Tucker, il suffit de calculer pour tout $i=1, \ldots, n$, en utilisant le logiciel PEPER, les nombres $C L_{i}$ de chiffres décimaux significatifs de chaque composante de grad $L(X, \lambda, \mu)$, avec :

$$
L(X, \lambda, \mu)=F(X)+\sum_{i=1}^{m} \mu_{i} H_{i}(X)-\sum_{j=m+1}^{p} \lambda_{j} G_{j}(X) .
$$

Aussi, si pour tout $i$ on a :

$$
C L_{i}<1, \quad i=1, \ldots, n,
$$

alors $X^{(q)}=X_{s}$ est une solution informatiquement satisfaisante dans la mesure où elle satisfait aussi les contraintes.

Pour vérifier les contraintes, il suffit de calculer le nombre de chiffres décimaux significatifs de chacune d'elles au point $X_{s}$ et de s'assurer que pour 
les contraintes d'égalité ces nombres sont tous inférieurs à 1 et pour les contraintes d'inégalité, que ces nombres sont supérieurs à 1 .

Remarquons que lorsque les contraintes sont linéaires, on doit pour simplifier utiliser les résidus normés (voir première partie). Aussi, le test d'arrêt optimal permet d'arrêter le processus itératif dès que toutes les relations (14) (première partie) sont vérifiées, c'est-à-dire dès qu'une solution informatiquement satisfaisante est atteinte.

\section{ESTIMATION DE LA PRECISION DE LA SOLUTION}

Lorsqu'un code d'optimisation contrainte ou non contrainte nous a fourni une solution $X_{s}$ informatiquement satisfaisante, cela ne veut pas dire pour autant qu'elle représente une bonne approximation de la solution mathématique.

En effet, donnons de ceci une interprétation géométrique très simple sur le problème élémentaire suivant :

Trouver $x^{*}$ tel que :

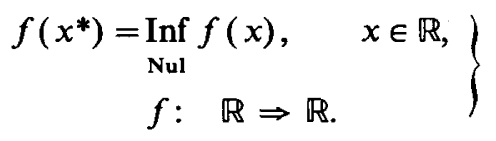

Comme il est montré dans la figure 1 , chacun des points $X \in \mathbb{F}, X \in\left[X_{1}, X_{2}\right]$ est une solution informatique $X_{s}$ satisfaisante de $F(X)=0$.

La grandeur de l'intervalle $I_{s}=\left[X_{1}, X_{2}\right]$ nous permet d'évaluer le nombre de chiffres décimaux significatifs de $X_{s} \in \mathbb{F}$. Plus $I_{s}$ est grand, moins $X_{s}$ approxime précisément $x^{*} \in \mathbb{R}$.

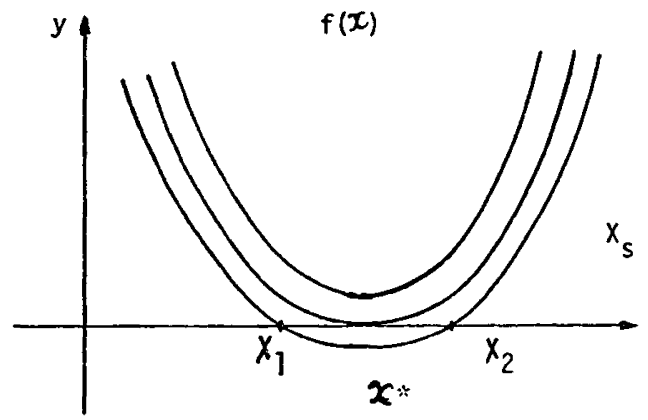

Fig. 1

Pour évaluer la précision de $X_{s} \in \mathbb{F}$, il suffit de résoudre trois fois le problème d'optimisation. On obtient ainsi trois solutions informatiquement satisfaisantes : $X_{s}^{(1)}, X_{s}^{(2)}, X_{s}^{(3)}$. 
La meilleure approximation de $x^{*} \in \mathbb{R}$ est $\bar{X}$ moyenne des trois $X_{s}^{j}, j=1,2,3$. Avec ces trois résultats, il est aisé avec l'équation (26) (première partie) d'estimer le nombre de chiffres décimaux significatifs exact de $\bar{X}$.

Remarque. - Afin de minimiser le temps de calcul on peut, après avoir obtenu $X_{s}^{1}$, recommencer l'optimisation avec, comme nouveau point de départ, une approximation de $X_{s}^{1}$.

Le code donné en annexe met en œuvre cette méthode et permet donc de fournir la précision sur la solution de tout problème d'optimisation non contrainte.

\section{EXEMPLES NUMÉRIQUES}

\section{1. Problème d'optimisation non contrainte}

Nous présentons ici un exemple très simple qui permet de montrer très clairement les inconvénients de l'utilisation des tests d'arrêt classiques et l'intérêt de l'utilisation de la méthode proposée (test d'arrêt optimal et évaluation de la précision de la solution). Le logiciel correspondant à cet exemple est présenté en annexe.

Soit à résoudre le système non linéaire défini par (2) en déterminant le Inf Nul de :

$$
F=f_{1}^{2}+f_{2}^{2} .
$$

La fonction $F$ admet deux minima : l'un absolu, correspondant à la solution du système non linéaire considéré, l'autre relatif. Nous présentons dans le tableau I les résultats fournis par le code d'optimisation proposé en annexe.

TABleaU I

\begin{tabular}{|c|c|c|c|c|c|c|c|}
\hline$X_{1}^{0}$ & $X_{2}^{0}$ & $X_{1 s}$ & $X_{2 s}$ & $C X_{1 s}$ & $C X_{2 s}$ & $F$ & Conclusion \\
\hline-1. & 50. & $2.000 \ldots 0$ & $1.000 \ldots 0$ & 14 & 12 & $1.10^{-24}$ & $\begin{array}{c}X_{s} \text { est solution } \\
\text { du système linéaire }\end{array}$ \\
\hline-5. & 22. & -2.025386 & -2.61553 & 7 & 6 & 5.25954 & $\begin{array}{c}X_{s} \text { n'est pas solution } \\
\text { du système linéaire }\end{array}$ \\
\hline
\end{tabular}

La valeur de $F=10^{-24}$ a été trouvée non significative et donc doit être considérée comme un zéro, et par conséquent la solution trouvée est bien solution du système non linéaire considéré.

La valeur de $F=5.25954$ est significative et déterminée avec 12 chiffres décimaux exacts; elle ne correspond pas à un minimum nul. 
Nous avons résolu le même système non linéaire en multipliant $f_{1}$ et $f_{2}$ par $10^{-20}$. Les résultats obtenus sont présentés dans le tableau II.

TABLEAU II

\begin{tabular}{|c|c|c|c|c|c|c|c|}
\hline$X_{1}^{0}$ & $X_{2}^{0}$ & $X_{1 s}$ & $X_{2 s}$ & $C X_{1 s}$ & $C X_{2 s}$ & $F$ & Conclusion \\
\hline-1. & 50. & $2.000 \ldots 0$ & $1.000 \ldots 0$ & 14 & 12 & $2.10^{-64}$ & $\begin{array}{c}X_{s} \text { est solution } \\
\text { du systeme } \\
\text { non linéaire }\end{array}$ \\
\hline-5. & 22. & -2.025386 & -2.61553 & 7 & 6 & $5.10^{-40}$ & $\begin{array}{c}X_{s} \text { n'est pas solution } \\
\text { du système } \\
\text { non linéaire }\end{array}$ \\
\hline
\end{tabular}

Remarquons ici que le logiciel proposé a permis de conclure que la valeur $F=5.10^{-40}$ est bien significative et qu'elle est connue avec 13 chiffres décimaux significatifs. Ainsi, malgré la faible valeur de $F$, le logiciel a pu conclure que la solution $X_{1}=-2.025386 ; X_{2}=-2.61553$ n'est pas solution du système non linéaire.

Nous avons résolu le même système non linéaire en multipliant $f_{1}$ et $f_{2}$ par $10^{+30}$. Les résultats obtenus sont présentés dans le tableau III.

TABLEAU III

\begin{tabular}{|c|c|c|c|c|c|c|c|}
\hline$X_{1}^{0}$ & $X_{2}^{0}$ & $X_{1 s}$ & $X_{2 s}$ & $C X_{1 s}$ & $C X_{2 s}$ & $F$ & Conclusion \\
\hline-1. & 50. & $2.000 \ldots 0$ & $1.000 \ldots 0$ & 13 & 12 & $6.6 .10^{+36}$ & $\begin{array}{c}X_{s} \text { est solution } \\
\text { du système } \\
\text { non linéaire }\end{array}$ \\
\hline-5. & 22. & -2.025386 & -2.61553 & 7 & 6 & $5.2 .10^{+61}$ & $\begin{array}{c}X_{s} \text { n'est pas solution } \\
\text { du système } \\
\text { non linéaire }\end{array}$ \\
\hline
\end{tabular}

Remarquons ici que le logiciel proposé a permis de conclure que la valeur $F=6.6 \cdot 10^{+36}$ est non significative et ne représente que les erreurs de calculs; elle doit être considérée comme un zéro mathématique. Ainsi, malgré la valeur élevée de $F$, la solution $X_{1}=2, X_{2}=1$ est bien solution du système non linéaire.

Le logiciel de Permutation-Perturbation permet aussi d'évaluer l'influence des erreurs de données sur les résultats d'algorithmes numériques.

En effet il suffit, lors des trois résolutions du problème d'optimisation, de perturber les données en fonction de la précision avec laquelle elles sont connues.

Aussi nous avons résolu le même système linéaire en supposant que les valeurs des constantes qui figurent dans les fonctions $f_{1}$ et $f_{2}$ sont connues vol. $18, \mathrm{n}^{\circ} 2$, mai 1984 
avec une précision relative de $10^{-5}$. Les résultats obtenus sont présentés dans le tableau IV.

TABleaU IV

\begin{tabular}{|c|c|c|c|c|c|c|c|}
\hline$X_{1}^{0}$ & $X_{2}^{0}$ & $X_{1 s}$ & $X_{2 s}$ & $C X_{1 s}$ & $C X_{2 s}$ & $F$ & Conclusion \\
\hline-1. & 50. & 2.000 & 1.00 & 4 & 3 & $7.2 .10^{-26}$ & $\begin{array}{c}X_{s} \text { est solution } \\
\text { du système } \\
\text { non linéaire }\end{array}$ \\
\hline-5. & 22. & -2.025 & -2.615 & 4 & 4 & 5.2 & $\begin{array}{c}X_{s} \text { n'est pas solution } \\
\text { du système } \\
\text { non linéaire }\end{array}$ \\
\hline
\end{tabular}

Nous voyons ici que compte tenu des erreurs sur les données, $X_{1}$ et $X_{2}$ ne peuvent être déterminés qu'avec 3 ou 4 chiffres décimaux significatifs.

Ces exemples mettent bien en évidence que le seul test d'arrêt efficace est celui proposé ici.

Le logiciel que nous proposons et qui est donné en annexe nous permet donc d'arrêter le processus itératif dès qu'une solution informatiquement satisfaisante est atteinte, il minimise ainsi le nombre des itérations et, de plus, il fournit la précision de la solution.

\section{V.2. Problème d'optimisation contrainte}

Nous présentons ici un exemple très simple d'optimisation contrainte qui met en évidence l'efficacité de la méthode proposée pour vérifier les contraintes et la solution trouvée.

Soit à déterminer :

$$
\operatorname{Inf} F(X)=\left(X_{1}-2\right)^{2}+\left(X_{2}-1\right)^{2},
$$

sous les contraintes :

$$
\begin{gathered}
h_{1}(X)=X_{1}-2 X_{2}+1=0, \\
g_{1}(X)=\frac{-X_{1}^{2}}{4}-X_{2}^{2}+1 \geqq 0 .
\end{gathered}
$$

D'après ce qu'il a été dit précédemment, nous avons transformé la contrainte d'inégalité (19) en contrainte d'égalité (20) définie par :

$$
\left.\begin{array}{c}
g_{1}(X)=\frac{-X_{1}^{2}}{4}-X_{2}^{2}+1-X_{3}=0, \\
X_{3} \geqq 0 .
\end{array}\right\}
$$

R.A.I.R.O. Recherche opérationnelle/Operations Research 
Les conditions de Kuhn et Tucker s'expriment par :

$$
\begin{gathered}
\mathrm{C} 1=2\left(X_{1}+2\right)+\mu+\frac{\lambda X_{1}}{2}=0, \\
\mathrm{C} 2=2\left(X_{2}-1\right)-2 \mu+2 \lambda X_{2}=0, \\
\mathrm{C} 3=X_{1}-2 X_{2}+1=0, \\
\mathrm{C} 4=\frac{-X_{1}^{2}}{4}-X_{2}^{2}+1-X_{3}=0, \\
\mathrm{C} 5=\lambda\left(-\frac{X_{1}^{2}}{4}-X_{2}^{2}+1\right)=0, \\
X_{3} \geqq 0, \quad \mu \geqq 0 .
\end{gathered}
$$

Nous avons résolu ce problème en utilisant le logiciel de PermutationPerturbation sur CDC 7600. Nous avons obtenu trois solutions informatiquement satisfaisantes qui vérifient les contraintes et les conditions de Kuhn et Tucker.

$\mathrm{C} 1$ et $\mathrm{C} 2$ sont vérifiées lorsque leurs nombres de chiffres significatifs NC 1 et NC 2 sont tous deux inférieurs à 1 .

C 3 et C 4 étant linéaires, on les vérifie par la méthode des résidus normés exposés dans la première partie. Si $\rho_{\mathrm{C} 3}^{*}$ et $\rho_{\mathrm{C} 4}^{*}$ sont tous deux inférieurs à 1 , alors les contraintes $\mathrm{C} 3$ et $\mathrm{C} 4$ sont satisfaites.

Les résultats obtenus sont présentés dans le Tableau V.

TABLEAU V

\begin{tabular}{|c|c|c|c|}
\hline & $1^{\text {re }}$ résolution & $2^{\mathrm{e}}$ résolution & $3^{\mathrm{e}}$ résolution \\
\hline $\mathrm{X} 1$ & 0.822875655532297 & 0.822875655532293 & 0.822875655532297 \\
\hline $\mathrm{X} 2$ & 0.911437827766147 & 0.911437827766146 & 0.911437827766147 \\
\hline $\mathrm{X} 3$ & $-1.77635683940026 .10^{-14}$ & 0. & $1.77635683940025 .10^{-14}$ \\
\hline$\mu$ & 1.59449111825231 & 1.59449111825232 & 1.59449111825231 \\
\hline$\lambda$ & 1.84659143960610 & 1.846591143960610 & 1.84659143960610 \\
\hline $\mathrm{NC} 1$ & 0. & 0. & 0. \\
\hline $\mathrm{NC} 2$ & 0. & 0. & 0. \\
\hline$\rho_{\mathrm{C} 3}^{*}$ & 0. & 0. & 0.1 \\
\hline$\rho_{\mathrm{C} 4}^{*}$ & 0.1 & 0.1 & 0. \\
\hline
\end{tabular}


Avec les trois valeurs des inconnues, il est aisé de calculer leur nombre de chiffres décimaux significatifs exacts, comme nous l'avons expliqué précédemment.

En appelant respectivement NCX 1, NCX 2, NCX 3, NC $\mu, N C \lambda$, le nombre de chiffres décimaux significatifs exacts de X1, X $2, X 3, \mu$ et $\lambda$, nous obtenons :

$$
\begin{aligned}
& \operatorname{NCX} 1=14, \\
& \operatorname{NCX} 2=14, \\
& \operatorname{NCX} 3=0, \\
& \operatorname{NC} \mu=14, \\
& \operatorname{NC} \lambda=15 .
\end{aligned}
$$

Nous tombons ici sur le cas particulier où l'un des nombres significatifs, NCX 3, est nul. Ou bien X 3 ne peut pas être calculé car la précision de l'ordinateur est trop faible, ou bien $\mathrm{X} 3=0$. Pour lever cette indétermination, il suffit de vérifier la contrainte $C 4$ avec les valeurs de $X 1, X 2, \mu, \lambda$ trouvées et $\mathrm{X} 3=0$.

Pour ces valeurs, on obtient : $\rho_{\mathrm{C} 4}^{*}=0.5$. La contrainte est donc satisfaite. Par conséquent, la solution est :

$$
\begin{gathered}
\text { X } 1=0.82287565553229, \quad \text { NCX } 1=14, \\
X 2=0.91143782776614, \quad \text { NCX } 2=14, \\
\text { X 3 }=0 . \quad \text { NCX } 3=15, \quad \mu=1.59449111822523, \quad \text { NC } \mu=14, \\
\lambda=1.84659143960610, \quad \text { NC } \lambda=75 .
\end{gathered}
$$

\section{CONCLUSION}

Nous avons présenté dans cet article une application de la méthode de Permutation-Perturbation aux algorithmes d'optimisation.

L'utilisation de cette méthode permet, pour les méthodes d'optimisation contraintes ou non contraintes, d'arrêter le processus itératif dès qu'une solution informatique satisfaisante est atteinte et d'estimer la précision de cette solution.

En outre, cette méthode permet de tenir compte des erreurs sur les données du problème.

La méthode de Permutation-Perturbațion permet donc de faire faire automatiquement à l'ordinateur l'analyse de la propagation des erreurs dues à l'arithmétique à précision limitée de la machine, ainsi que l'estimation des conséquences des erreurs de données sur les résultats de toute optimisation.

R.A.I.R.O. Recherche opérationnelle/Operations Research 
Le logiciel tel qu'il est proposé ici fournit donc pour tout problème d'optimisation le résultat avec sa précision associée. Ce problème n'avait jamais été résolu jusqu'ici.

\section{BIBLIOGRAPHIE}

1. J. AbAdie, The GRG Method for Nonlinear Programming Design and Implementation of Optimization Software. In: Harvey J. Greenberg, éd., Sijthoff \& Noordhoff, 1978, p. 335-362.

2. D. M. Himmelblau, Numerical Methods for Nonlinear Optimization. In: F. A. Lootsma, éd., Academic Press, 1972, p. 69-73.

3. M. LA PORTE et J. Vignes, Algorithmes numériques, analyse et mise en auvre, tome 1, Technip, ed., Paris, 1974.

4. M. LA Porte et J. Vignes, Étude statistique des erreurs dans l'arithmétique des ordinateurs; application au contrôle des résultats d'algorithmes numériques, Numer. Math., vol. 23, 1974, p. 63-72.

5. M. MAILlÉ, Some Methods to Estimate Accuracy of Measures or Numerical Computations. Proceedings of Mathematics for Computer Science, Colloque international AFCET, Paris, 1982, p. 495-503.

6. P. Tolla, Stabilisation et accélération d'algorithmes de Programmation mathématique: Gradient Réduit Généralisé et Moindres Carrés, Actes du Symposium AFCET "Les Mathématiques pour l'Informatique ", Paris, 1982.

7. P. Tolla, Linear and Nonlinear Programming Software Validity. Math. and Comp. in Sim., vol. XXV, 1983, p. 39-42.

8. J. Vignes et M. LA Porte, Error Analysis in Computing. Proceedings of IFIP Congress, Stockholm, 1974, p. 609-614.

9. J. VIGNES, Étude et mise en æuvre d'algorithmes de recherche d'un extremum d'une fonction de plusieurs variables. Thèse d'État, Paris, 1969.

10. J. VIGNes, New Methods for Evaluating the Validity of the Results of Mathematical Computations. Math. and Comp. in Sim. Vol. XX, $\mathrm{n}^{\circ} 4$, 1978, p. 227-249. 


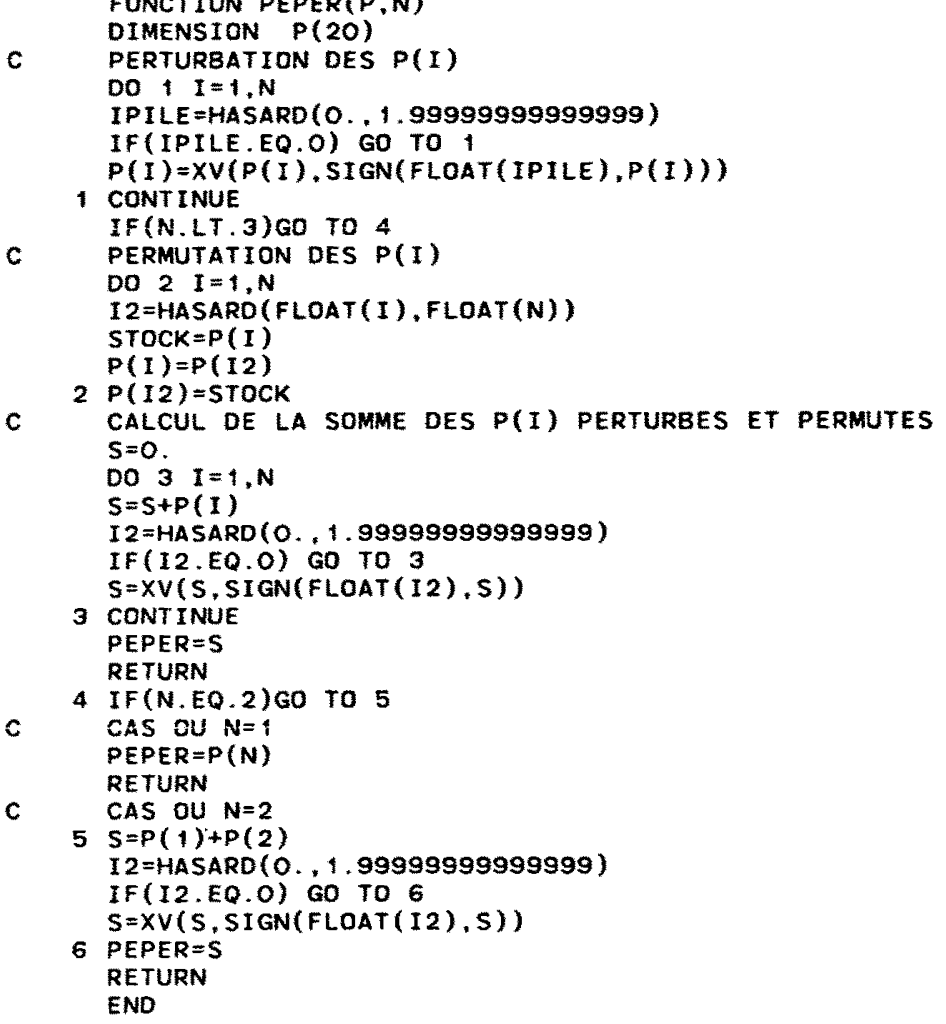

FUNCTION $X V(X, S)$

$\operatorname{PRE}(Z)=$ OR $(00004000000000000000 B, \operatorname{AND}(\operatorname{ABS}(Z), 77770000000000000000 \mathrm{~B}))$ DER $(Z)=0 R(00000000000000000001 B, A N D(A B S(Z), 77770000000000000000 B))$ IF (X.EQ.O. GO TO 1 $X V=X+S I G N(D E R(X), S)$

Remarque : La fonction PEPER fait appel à une fonction Hasard qui génère aléatoirement les valeurs 0 et 1 . 


\section{ANNEXES}

Texte du sous-programme FORTRAN de résolution d'un système d'équations non linéaire par la méthode d'optimisation avec test d'arrêt optimal et calcul de la précision de la solution.

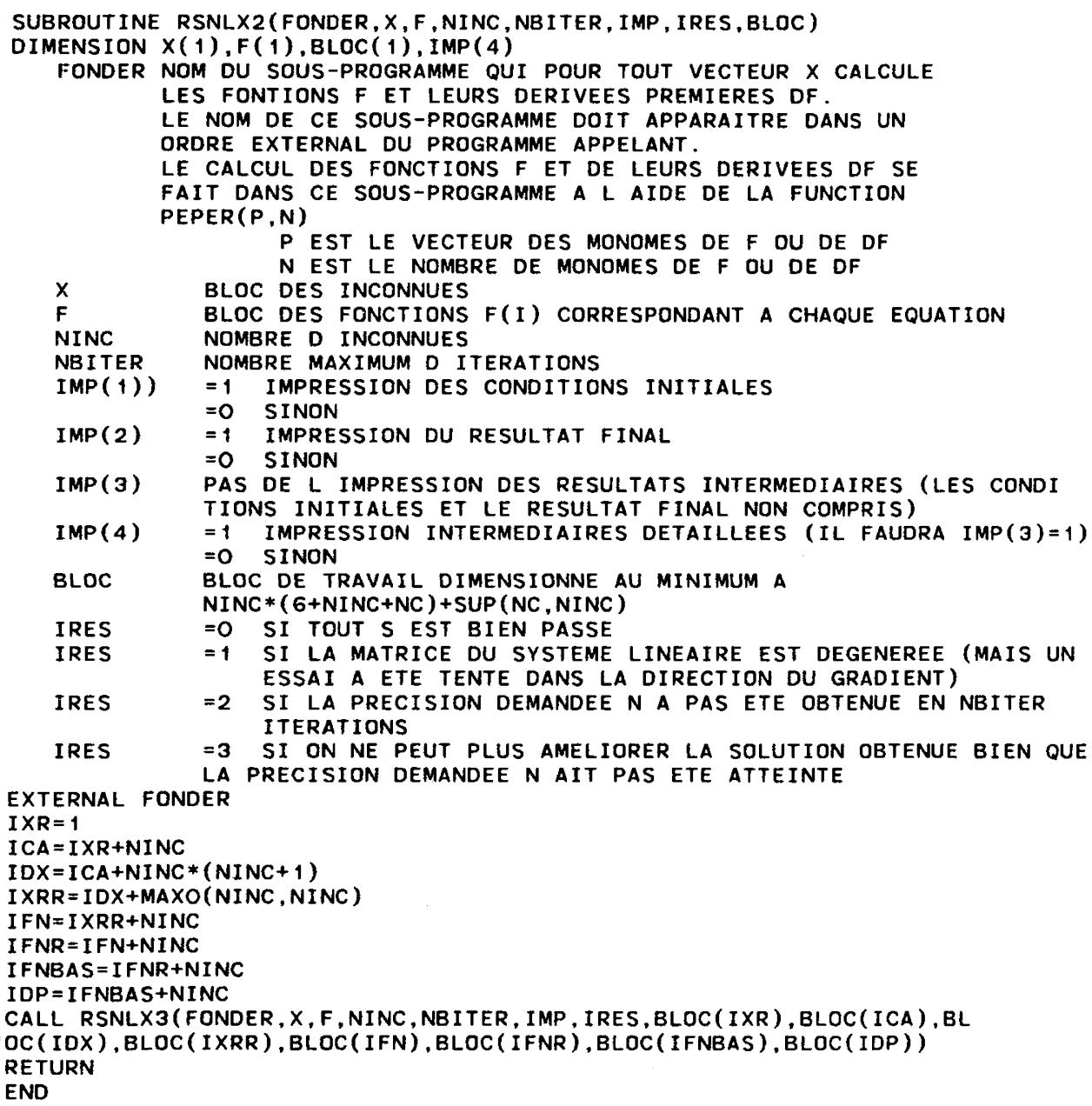


SUBROUT INE RSNLX3(FONDER, X, F, NINC, NBITER, IMP, IRES, XR, CA, DX, XRR, FN, 1FNR, FNBAS, DP)

DIMENSION $X(1), F(1), \quad \operatorname{IMP}(5)$

DIMENSION XR( 1$), \operatorname{CA}(N I N C, 1), \operatorname{DX}(1), \operatorname{XRR}(1), \operatorname{FN}(1), \operatorname{FNR}(1), \operatorname{FNBAS}(1), \operatorname{DP}(N$

1INC , 1)

DIMENSION FSTOK $(20,3)$

EXTERNAL FONDER

DIMENSION DF2 (10), FPP $(10,5), F M(10), \operatorname{VAR}(10), \operatorname{ERM}(10), C(10)$

DIMENSION XCRESM(10), XCRES $(10,5), \operatorname{XCVAR}(10), \operatorname{ERMXC}(10), \operatorname{CXC}(10)$

DIMENSION CXCRES $(10,5), X O(10)$

100 FORMAT ( $1 \mathrm{H} 1)$

101 FORMAT $(/ / / /)$

102 FORMAT(5X, *SOLUTION OBTENUE PAR LA DIRECTION DU SYSTEME LINEAIRE*/ $1 / /)$

103 FORMAT(5X, *SOLUTION OBTENUE PAR LA DIRECTION DU GRADIENT*///)

104 FORMAT(5X,*SOLUTION OBTENUE PAR LA DIRECTION DE LA BISSECTRICE*/// 1)

105 FORMAT $(/ / 30 X, *$ SYSTEME LINEAIRE*)

106 FORMAT $(30 X, * E Q=*, E 22.15)$

107 FORMAT (/, 3OX, *SOLUTION DANS LA DIRECTION DONNEE PAR LE SYSTEME LIN 1EAIRE*)

108 FORMAT (3OX, $2 \mathrm{HX}(, \mathrm{I} 2,2 \mathrm{H})=, \mathrm{E} 22.15)$

109 FORMAT (//30X,*BISSECTRICE DES DEUX PRECEDENTES OIRECTIONS DU GRADI IENT*)

110 FORMAT (//30X,*GRADIENT APRES SUCCES SYSTEME LINEAIRE*)

111 FORMAT (//3OX,*BISSECTRICE APRES SUCCES SYSTEME LINEAIRE*)

112 FORMAT (/3OX, *SOLUTION DANS LA DIRECTION DU GRADIENT*)

113 FORMAT (/3OX, *SOLUTION DANS LA DIRECTION DE LA BISSECTRICE*)

114 FORMAT (//3OX, *GRADIENT APRES ECHEC SYSTEME LINEAIRE*)

115 FORMAT (//30X, *BISSECTRICE APRES ECHEC SYSTEME LINEAIRE*)

116 FORMAT (/3OX, *SOLUTION DANS LA DIRECTION DU GRADIENT*)

117 FORMAT (/3OX, *SOLUTION DANS LA DIRECTION DE LA BISSECTRICE*)

118 FORMAT $(1 \mathrm{H}, / / /$ SOX, 17HMATRICE DEGENEREE, ///)

119 FORMAT $(1 \mathrm{H}, / / /$ 5OX,34HPRECISION DEMANDEE NON OBTENUE EN , I3, 112H ITERATIONS.///)

120 FORMAT $(/ / / 40 X, *$ LA SOLUTION OBTENUE a L ITERATION*,13,* NE PEUT PLU 1S ETRE AMELIOREE*)

121 FORMAT $(5 X, 2 H X(, 12,4 H)=, E 22.15)$

122 FORMAT $(/ 5 X, 3$ HEQ $(.13,4 H)=. E 22.15)$

NC $=$ NINC

$I$ COMPT $=0$

$J C O M P T=0$

DO $70 I=1, N I N C$

$70 X O(I)=X(I)$

$71 \mathrm{~J}=0$

DO $72 \quad I=1$, NINC

$72 X(I)=X O(I)$

IRES $=0$

NM $1=N I N C-1$

$N N=N I N C+1$

$\mathrm{N}=\mathrm{O}$

$\operatorname{IF}(\operatorname{IMP}(3) \cdot E Q \cdot 0) \operatorname{IMP}(3)=N B \operatorname{ITER}+2$

CALL FONDER(X,NINC, F,DP, 3)

$E Q=0$.

DO $1 \mathrm{I}=1, \mathrm{NC}$

$1 E Q=E Q+F(I) * * 2$

EQBAS $=E Q$

$\operatorname{IMP} 1=\operatorname{IMP}(1)+\operatorname{IMP}(2)+\operatorname{IMP}(3)+\operatorname{IMP}(4)$

IF (IMP 1. NE . O)PRINT 100

$\operatorname{IF}(\operatorname{IMP}(1)$.EQ.0)GO TO 8

PRINT 121, $(I, X(I), I=1$, NINC )

PRINT 122,N,EOBAS

PRINT 101

GO TO 8

R.A.I.R.O. Recherche opérationnelle/Operations Research 
C

TEST D ARRET OPTIMAL

$2 J J=J U+1$

CALL FONDER (X,NINC, F,DP, 3)

DO $1000 I=1$, NINC

DF 2 (I) $=0$.

DO $1000 K=1$, NINC

1000 DF 2 (I ) $=D F 2(I)+2 * F(K) * D P(K, I)$

DO $531 I=1$, NINC

$\operatorname{FPP}(I, J U)=D F 2(I)$

531 CONTINUE

IF ( JU.LT.3)GO TO 2

C CALCUL DES C(F) DES DF 2 (I)

C CALCUL DES DF2 (F) MOYENS

DO $534 I=1$, NINC

$F M(I)=0$.

DO $534 K=1, \mathrm{~J}$

$534 F M(I)=F M(I)+F P P(I . K)$

DO $535 I=1$, NINC

$535 F M(I)=F M(I) / F L O A T(J J)$

c

CALCUL DES VARIANCES DES DF2(I)

DO $536 I=1$, NINC

$\operatorname{VAR}(I)=0$.

DO $536 K=1$, J J

$536 \operatorname{VAR}(I)=\operatorname{VAR}(I)+($ FPP $(I, K)-F M(I)) * * 2$

DO $537 I=1$, NINC

$537 \operatorname{VAR}($ I $)=\operatorname{VAR}(\mathrm{I}) /$ FLOAT $(\mathrm{JJ}-1)$

C

CALCUL DE L ERREUR MOYENNE ERM(I)

DO $538 \quad I=1$, NINC

c

$538 \operatorname{ERM}(I)=\operatorname{SQRT}((\operatorname{FPP}(I, 1)-F M(I)) * * 2+\operatorname{VAR}(I))$

CALCUL DU C(I) DES DF2(I)

DO $539 I=1$, NINC

IF (ERM (I).EQ.O.) GO TO 540

$G=A B S(F P P(I, 1) / E R M(I))$

IF (G.EQ.O.) GO TO 541

$C(I)=A L O G 10(G)$

GO TO 539

$541 C(I)=0$.

GO TO 539

$540 \mathrm{C}(\mathrm{I})=15$.

IF (VAR (I) EE.O. )C (I ) $=0$.

539 CONTINUE

IF(IT1.EQ.5O) GO TO 733

DO $507 I=1$, NINC

IF (C (I).GE. 1.) GO TO 4

507 CONTINUE

C

CALCUL DU NOMBRE DE CHIFFRES SIGNIFICATIFS DE LA SOLUTION

C

733 I COMPT $=I$ COMPT +1

IF (IMP (3).EQ.O) GO TO 334

PRINT 199, ICOMPT

199 FORMAT( 1 H, * SOLUTION TROUVEE A LA*, 12,* IEME RESOLUTION*) DO $333 K=1$, NINC

333 PRINT $123, K, X(K)$

123 FORMAT $(21 X, 2 H X(, I 2,2 H)=, E 22,15)$

PRINT 200, EQBAS

200 FORMAT $(21 X, * E Q=*, E 22.15)$

334 DO $550 \quad I=1$, NINC

$550 \times \operatorname{CRES}(I, I$ COMPT $)=X(I)$

IF (ICOMPT.LT.2)GO TO 71

C CALCUL DES $X$ (I) MOYENS

DO $551 I=1$, NINC

$X \operatorname{CRESM}(I)=0$.

DO $551 K=1$, I COMPT

$551 \operatorname{XCRESM}(I)=\operatorname{XCRESM}(I)+\operatorname{XCRES}(I, K)$

vol. $18, \mathrm{n}^{\circ} 2$, mai 1984 
DO $552 I=1, N I N C$

$552 \operatorname{XCRESM}(I)=X C R E S M(I) / F L O A T$ ( ICOMPT)

C CALCUL DE LA VARIANCE DU VECTEUR SOLUTION DO $553 I=1$, NINC $\operatorname{XCVAR}(I)=0$. DO $553 K=1$, I COMPT

$553 \operatorname{XCVAR}(I)=\operatorname{XCVAR}(I)+(\operatorname{XCRES}(I, K)-\operatorname{XCRESM}(I)) * * 2$ DO $554 I=1$, NINC

C

$554 \times \operatorname{XVVAR}(I)=X C V A R(I) / F L O A T(I$ COMPT -1$)$ CALCUL DE L ERREUR MOYENNE SUR LE VECTEUR SOLUTION DO $555 I=1$, NINC

555 ERMXC(I) $=$ SORT ( (XCRE
CALCUL DES CXC (I)

DO $556 \quad I=1$, NINC

IF (ERMXC (I).EQ.O.)GO TO 557

G2 $=A B S(X C R E S(1,1) / E R M X C(I))$

IF (G2.EQ.O.) GO TO 558

$\mathrm{CXC}(\mathrm{I})=A \operatorname{LOG} 10(\mathrm{G2})$

GO TO 556

$557 \mathrm{CXC}(\mathrm{I})=15$.

IF (XCVAR (I) EQ E. O. ) CXC (I) $=0$.

GO TO 556

$558 \operatorname{CXC}(1)=0$.

556 CONTINUE

C

MISE A ZERO DES XC(I) DONT LE CXC(I) EST INFERIEUR A 1 DO $570 \quad I=1$, NINC

IF (CXC(I).LT.1.) GO TO 571

GO TO 570

$571 \times(I)=0$.

$\operatorname{CXC}(\mathrm{I})=15$.

570 CONTINUE

C STOCKAGE DES CXC(I) $J C O M P T=J C O M P T+1$

DO $559 I=1$, NINC

$559 \operatorname{CXCRES}(I$, JCOMPT $)=\operatorname{CXC}(\mathrm{I})$

IF ( UCOMPT.GE.5) GO TO 5000

IF ( JCOMPT.EQ.1)GO TO 71

DO $561 \mathrm{I}=1$, NINC

IF (CXCRES (I, JCOMPT)-CXCRES (I, JCOMPT-1).GT .0.5)GO TO 71

561 CONTINUE

IMPRESSION DE LA PRECISION DU VECTEUR SOLUTION

PRINT 124

124 FORMAT ( 1 HO , 4X, *SOLUTION FINALE TROUVEE*)

DO $509 K=1$, NINC

$I C=\operatorname{CXCRES}(K, J C O M P T)+0.5$

509 PRINT 125,K, IC+7,IC, $X(K), 20-I C, I C$

125 FORMAT $(6 X, * X(*, I 3, *)=*, E=, *,=X, * C=*, 13)$

DO $900 L=1.3$

CALL FONDER(X,NINC, F, DP, 1)

DO $900 \quad I=1$, NINC

c

$900 \operatorname{FSTOK}(I, L)=F(I)$

DO $901 I=1$, NINC

$F M(I)=0$.

DO $901 L=1,3$

$901 F M(I)=F M(I)+F S T O K(I, L)$

DO $902 I=1$, NINC

c

$902 F M(I)=F M(I) / 3$.

DO $903 I=1$, NINC

$\operatorname{VAR}(I)=0$.

DO $903 \mathrm{~L}=1,3$

$903 \operatorname{VAR}(I)=\operatorname{VAR}(I)+(\operatorname{FSTOK}(I, L)-F M(\zeta)) * * 2$

R.A.I.R.O. Recherche opérationnelle/Operations Research 
DO $904 \mathrm{I}=1$, NINC

c

$904 \operatorname{VAR}(I)=\operatorname{VAR}(I) / 2$

c

DO $905 \quad I=1$, NINC

$905 \operatorname{ERM}(1)=\operatorname{SQRT}((\operatorname{FSTOK}(I, 1)-\mathrm{FM}(I)) * * 2+\operatorname{VAR}(I))$

DO $939 I=1$, NINC

IF (ERM(I).EQ.O.)GO TO 940

$G=A B S(F S T O K(I, 1)) / E R M(I)$

IF (G.EQ.O.) GO TO 941

$C(I)=A L O G 1 O(G)$

GO TO 939

$941 C($ I $)=0$.

Go TO 939

$940 C(I)=15$

IF $(\operatorname{VAR}(I), E Q .0) C.(I)=0$.

939 CONTINUE

DO $942 \quad I=1$, NINC

IF (C(I).GT.1.)GO TO 951

942 CONTINUE

PRINT 8500

8500 FORMAT $(6 \mathrm{X}$ *LA SOLUTION TROUVEE EST SOLUTION DU SYSTEME NON LINEAIR 1E*)

DO $952 I=1, N I N C$

$952 F(I)=0$.

PRINT $8503,(I, F(I), I=1, N I N C)$

8503 FORMAT $(40 X, * F(*, I 3, *)=*, F 3.0)$

RETURN

951 PRINT 8501

8501 FORMAT(6X,*LA SOLUTION TROUVEE N EST PAS SOLUTION DU SYSTEME NON L 1 INEAIRE CONSIDERE*)

DO $953 I=1$, NINC

I $C=C(I)+0.5$

953 PRINT 8502,I,IC+7, IC, F(I ), 20-IC, IC

$8502 \operatorname{FORMAT}(40 X, * F(*, 13, *)=*, E=.=,=X, * C=*, 13)$

RETURN

5000 PRINT 126

126 FORMAT( HHO. 4X, *PRECISION DE LA SOLUTION NON TROUVEE APRES 5 RUNS*) RETURN

$4 \mathrm{~J} J=0$ DO $5 I=1, N C$

$5 F(I)=D \times(I)$

CALL FONOER(X,NINC, F,DP, 2)

IF (MOD(N, IMP (3)).NE.O)GO TO 8

PRINT 121, $(I, X(I), I=1, N I N C)$

PRINT 122,N,EQBAS

IF (IPASS. EQ. 1)GO TO 6

IF (IPASS.EQ. 2 ) GO TO 7

PRINT 104

GO TO 8

6 PRINT 102

GO TO 8

7 PRINT 103

C

$8 \mathrm{~N}=\mathrm{N}+1$

$Y 1=E Q B A S$

$I B I=0$

IF (N.GT.NBITER) GO TO 64

C RANGEMENT DES FONCTIONS DANS LA MATRICE DU SYSTEME LINEAIRE.

C

DO $10 \mathrm{I}=1, \mathrm{NINC}$

$X R(I)=X(I)$

$\mathrm{CA}(I, N N)=0$.

DO $9 \quad J=Y, N C$

$9 C A(I, N N)=C A(I, N N)-F(J) * D P(U, I)^{\sim}$

$10 \mathrm{FN}(\mathrm{I})=-\mathrm{CA}(\mathrm{I}, \mathrm{NN})$ 


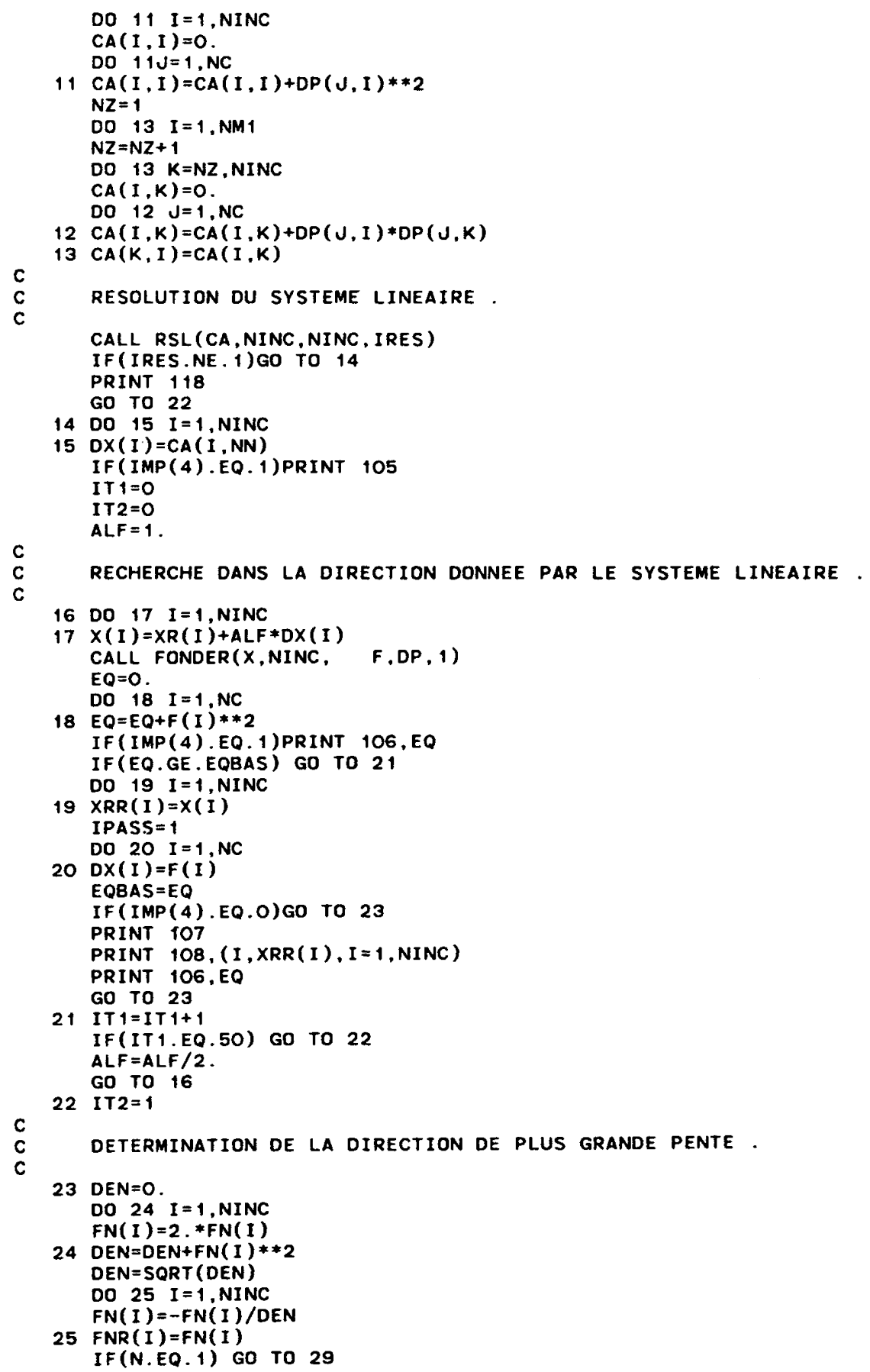


CALCUL DU COSINUS DE L"ANGLE DE DEUX DIRECTIONS SUCCESSIVES DE PLUS GRANDE PENTE

COTETA $=0$.

DO $26 I=1$, NINC

26 COTETA $=$ COTETA+FN(I)*FNBAS(I)

IF (COTETA.GT.O.) GO TO 29

$I B I=1$

IF(IMP (4) .EQ, 1)PRINT 109

C

C

CALCUL DE LA BISSECTRICE DES DEUX PRECEDENTES OIRECTIONS

C

$D E N=0$.

DO $27 I=1$, NINC

$F N(I)=F N(I)+F N B A S(I)$

27 DEN=DEN+FN(I)**2

DEN $=$ SQRT (DEN)

DO $28 I=1$, NINC

$28 F N(I)=F N(I) / D E N$

29 IF (IT2.EQ.1) GO TO 47

c

c

c

RECHERCHE DANS LA DIRECTION DU GRADIENT (OU BISSECTRICE)

APRES SUCCES DANS LA DIRECTION DONNEE PAR LE SYSTEME LINEAIRE

$I T 1=0$

IF (IMP (4).EO.0)GO To 30

IF (IBI.EQ.O)PRINT 110

IF (IBI.EQ.1)PRINT 111

$30 \mathrm{~s}=0$.

DO $31 \mathrm{I}=1$, NINC

$S=S+(X(I)-X R(I)) * * 2$

31 FNBAS (I) $=$ FNR (I)

$A L F=\operatorname{SORT}(S)$

32 DO $33 I=1$, NINC

$33 \times(I)=X R(I)+A L F * F N(I)$

CALL FONDER $(X, N I N C, \quad F, D P, 1)$

$E Q=0$.

DO $34 I=1, N C$

$34 E Q=E Q+F(I) * * 2$

IF(IMP(4).EQ. 1)PRINT 106, EQ

IF (EQ.GT. EQBAS)GO TO 38

IF (EQ.LT . EQBAS)GO TO 35

IF (IT1.EQ.O)GO TO 38

35 DO $36 \quad I=1$, NINC

$36 X R R(I)=X(I)$

DO $37 \quad I=1, N C$

$37 \mathrm{DX}(\mathrm{I})=\mathrm{F}(\mathrm{I})$

EQBAS $=E Q$

IF (IT1.NE. O)GO TO 40

$A L F=A L F * 2$

GO TO 32

$38 \quad A L F=A L F / 2$.

$Y 3=E Q$

IT $1=I T 1+1$

IF (ITI.LE. S) GO TO 32

DO $39 I=1$, NINC

$39 X(I)=X R R(I)$

GO TO 2

40 IPASS $=2+I B I$

ALF $=A L F / 2, *(3, * Y 1-4, * E Q+Y 3) /(Y 1-2, * E Q+Y 3)$

DO $411=1$, NINC

$41 X(I)=X R(I)+A L F * F N(I)$

CALL FONDER(X,NINC, F,DP, 1)

vol. $18, \mathrm{n}^{\circ} 2$, mai 1984 
$E Q=0$.

DO $42 I=1, N C$

$42 E Q=E Q+F(I) * * 2$

IF (EQ.GT. EQBAS) GO TO 44

EQBAS $=E Q$

DO $43 \quad I=1, N C$

$43 \mathrm{DX}(\mathrm{I})=\mathrm{F}(\mathrm{I})$

GO TO 46

44 DO $45 I=1$, NINC

$45 X(I)=X R R(i)$

46 If (IMP(4). EQ.0)GO TO 2

IF (IBI.EQ.O)PRINT 112

IF(IBI.EQ.1)PRINT 113

PRINT 108, $(I, X(I), I=1, N I N C)$

PRINT 106, EQBAS GO TO 2

$c$
$c$

RECHERCHE DANS LA DIRECTION DU GRADIENT (OU BISSECTRICE) APRES ECHEC DANS LA DIRECTION DONNEE PAR LE SYSTEME LINEAIRE

47 IT $1=0$

IF (IMP (4) EQ.O)GO TO 48

IF (IBI.EQ. O)PRINT 114

IF ( IBI, EQ, 1)PRINT 115

48 DO $49 I=1$, NINC

49 FNBAS (I ) $=$ FNR (I)

$A L F=1$.

50 DO $51 I=1$, NINC

$51 X(I)=X R(I)+A L F * F N(I)$

CALL. FONDER(X,NINC, F,DP, 1)

$E Q=0$.

DO $52 I=1, N C$

$52 E Q=E Q+F(I) * * 2$

IF (IMP (4) .EQ. 1)PRINT 106, EQ

IF (EQ.GT. EQBAS) GO TO 56

IF (EQ.LT. EQBAS)GO TO 53

IF (IT1.EQ.O)GO TO 56

53 DO $54 I=1$, NINC

$54 X R R(I)=X(I)$

DO $55 I=1, N C$

$55 \mathrm{DX}(\mathrm{I})=\mathrm{F}(\mathrm{I})$

$E Q B A S=E Q$

IF (ITI.NE . O)GO TO 57

$A L F=A L F * 2$.

GO TO 50

56 IT $1=I T+1$

$Y 3=E Q$

IF(IT1.EO.5O) GO TO 65

$A L F=A L F / 2$.

GO TO 50

57 IPASS $=2+$ IBI

$A L F=A L F / 2, *(3, * Y 1-4, * E Q+Y 3) /(Y 1-2, * E O+Y 3)$

DO $58 I=1, N I N C$

$58 \times(I)=X R(I)+A L F * F N(I)$

CALL FONDER(X,NINC, F,DP, 1)

R.A.I.R.O. Recherche opérationnelle/Operations Research 


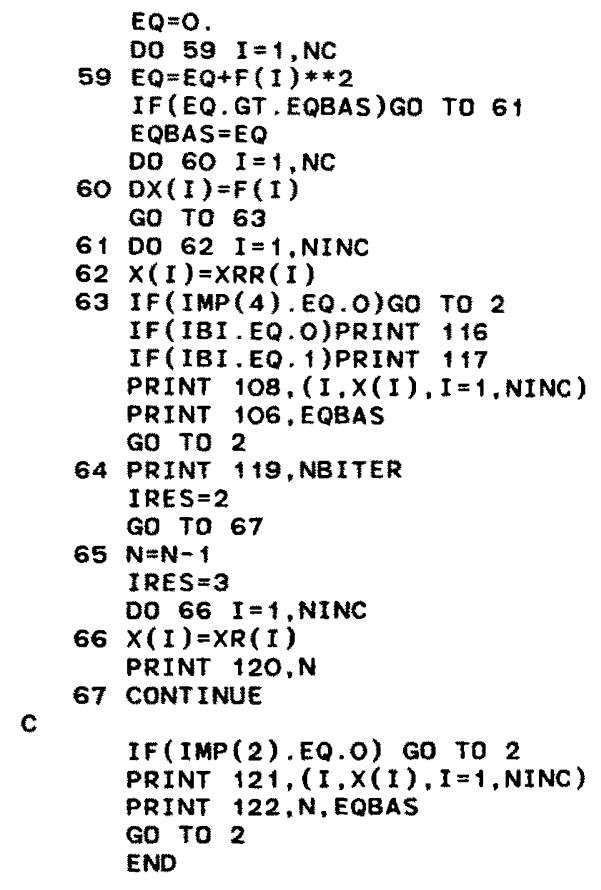

Remarque : Le sous-programme RSNLX 3 fait appel à un sous-programme RSL de résolution de systèmes d'équations linéaires. 
Exemple d'utilisation du sous-programme RSNLX 2.

Considérons le système d'ordre 2 suivant :

$$
\left\{\begin{array}{c}
f_{1}=7 x_{1}^{2}+3 x_{1} x_{2}+4 x_{1}-x_{2}-41=0 \\
f_{2}=10 x_{1}^{2}+4 x_{1} x_{2}+5 x_{1}-2 x_{2}-56=0
\end{array}\right.
$$

qui admet comme solution $x_{1}^{*}=2, x_{2}^{*}=1$.

Pour résoudre sur ordinateur ce système d'équations non linéaires par la méthode d'optimisation décrite précédemment avec test d'arrêt optimal et détermination de la précision sur la solution, on doit exprimer chacune des équations du système non linéaire et leurs dérivées partielles sous forme d'une suite de monômes dans un sous-programme. Les permutations-perturbations sont assurées par la fonction PEPER décrite précédemment.

(a) Programme principal

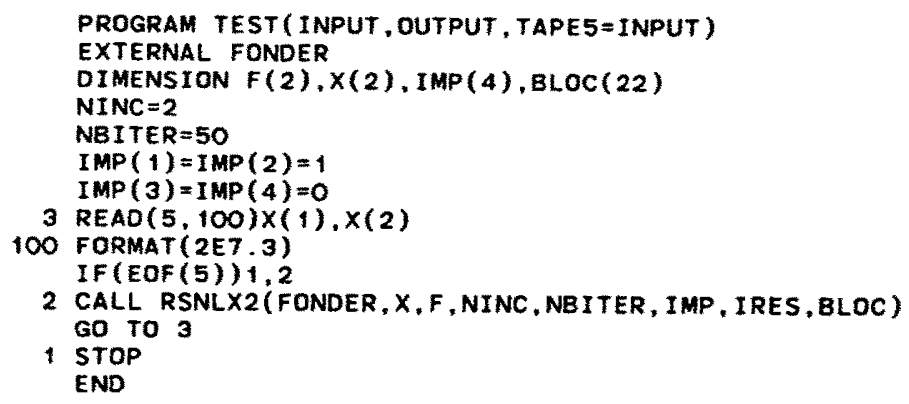

(b) Sous-programmeFONDER

Dans ce sous-programme sont exprimées, sous forme de suite de monômes, d'une part chacune des équations du système et d'autre part chacune des dérivées partielles des fonctions.

Notons que l'index ID nécessairement présent comme argument du sousprogramme permet de faire calculer par ce sous-programme, soit seulement les valeurs des fonctions ou des dérivées, soit l'ensemble des valeurs des fonctions et des dérivées. 
SUBROUTINE FONDER(X,NINC, F,DP, ID)

DIMENSION $X(1), F(1), D P(N I N C, 1), P(5)$

IF (ID.EQ.2) GO TO 10

$P(1)=7 . * x(1) * * 2$

$P(2)=3 . * x(1) * x(2)$

$P(3)=4 . * x(1)$

$P(4)=-X(2)-41$.

$F(1)=\operatorname{PEPER}(P, 4)$

$P(1)=10 * x(1) * * 2$

$P(2)=4, * x(1) * x(2)$

$P(3)=5 . * x(1)$

$P(4)=-2$.*X( 2) -56 .

$F(2)=\operatorname{PEPER}(P, 4)$

IF (ID.EQ.1) RETURN

to $P(1)=14 * * x(1)$

$P(2)=3 . * x(2)+4$.

$\operatorname{DP}(1,1)=\operatorname{PEPER}(P, 2)$

$P(1)=3 . * X(1)-1$.

$\operatorname{DP}(1,2)=\operatorname{PEPER}(P, 1)$

$P(1)=20 . * X(1)$

$P(2)=4 . * x(2)+5$.

$\operatorname{DP}(2,1)=\operatorname{PEPER}(\dot{P}, 2)$

$P(1)=4 . * X(1)-2$.

$\operatorname{DP}(2,2)=\operatorname{PEPER}(P, 1)$

RETURN

END

(c) Résultats obtenus sur CDC 7600

1.

$$
x_{1}^{0}=-1 \quad x_{2}^{0}=50
$$

$x(1)=-.100000000000000 E+01$

$x(2)=.500000000000000 E+02$

$E Q(0)=.179845000000002 E+06$

SOLUTION TROUVEE A LA 1 IEME RESOLUTION $X(1)=.199999999999999 E+01$ $X(2)=.999999999999950 E+00$ $E Q=.233937016987653 E-23$

$x(1)=-.100000000000000 E+01$

$x(2)=.500000000000000 E+02$

$E Q(0)=.179845000000001 E+06$

SOLUTION TROUVEE A LA 2 IEME RESOLUTION $X(1)=.200000000000009 E+01$ $X(2)=.999999999999346 E+\infty O$ $E Q=.516987882845642 E-25$

vol. $18, \mathrm{n}^{\circ} 2$, mai 1984 

$x(1)=-.100000000000000 E+01$
$x(2)=.500000000000000 E+02$
$E Q(0)=.179845000000000 E+06$

SOLUTION TROUVEE A LA 3 IEME RESOLUTION $X(1)=.200000000000001 E+01$

$X(2)=.999999999999758 E+\infty 0$

$\mathrm{EQ}=.103397576569128 \mathrm{E}-23$

SOLUTION FINALE TROUVEE

$X(1)=.20000000000000 E+01 \quad C=14$

$x(2)=.100000000000 E+01$

LA SOLUTION TROUVEE EST SOLUTION DU SYSTEME NON LINEAIRE $F(1)=0$.

$F(2)=0$.

2.

$$
x_{1}^{0}=-5 \quad x_{2}^{0}=22
$$

$x(1)=-.500000000000000 E+01$

$x(2)=.220000000000000 E+02$

$E Q(0)=.155869000000001 E+06$

$X(1)=-.202538615252618 E+01$

$X(2)=-.261552421503006 \mathrm{E}+01$

$E Q(20)=.525954133862228 E+01$

SOLUTION TROUVEE A LA 1 IEME RESOLUTION

$X(1)=-.202538615252618 E+01$

$X(2)=-.261552421503006 E+01$

$E Q=.525954133862228 E+01$

R.A.I.R.O. Recherche opérationnelle/Operations Research 
$x(1)=-.500000000000000 E+01$

$x(2)=.220000000000000 E+02$

$E Q(0)=.155868999999997 E+06$

$X(1)=-.202538566416861 E+01$

$X(2)=-.261552641352449 E+01$

$E Q(21)=.525954133862223 E+01$

SOLUTION TROUVEE A LA 2 IEME RESOLUTION

$X(1)=-.202538566416861 E+01$

$X(2)=-.261552641352449 E+01$

$E Q=.525954133862223 E+01$

$x(1)=-.500000000000000 E+01$
$X(2)=-.22000000000000 E+02$
$E Q(0)=. .155868999999998 E+06$

$X(1)=-.202538584795113 E+01$

$X(2)=-.261552555939312 \mathrm{E}+01$

EQ $(21)=.525954133862186 E+01$

SOLUTION TROUVEE A LA 3 IEME RESOLUTION

$X(1)=-.202538584795113 E+01$

$X(2)=-.261552555939312 E+01$

$E Q=.525954133862186 E+01$

SOLUTION FINALE TROUVEE

$\begin{array}{ll}X(1)=-.2025386 \mathrm{E}+01 & \mathrm{C}=7 \\ X(2)=-.261553 \mathrm{E}+01 & \mathrm{C}=6\end{array}$

LA SOLUTION TROUVEE N EST PAS SOLUTION DU SYSTEME NON LINEAIRE CONSIDERE

$F(1)=-.1878357641998 E+01 \quad C=13$

$F(2)=.1315794021635 E+01 \quad C=13$ 